\title{
Phase separation during film growth
}

\author{
M. Atzmon, a) D. A. Kessler, ${ }^{\text {b) }}$ and D. J. Srolovitz ${ }^{\text {() }}$ \\ University of Michigan, Ann Arbor, Michigan 48109
}

(Received 27 January 1992; accepted for publication 30 March 1992)

\begin{abstract}
A diffusion equation describing phase separation during co-deposition of a binary alloy is derived, and solved in the limit of dominant surface diffusion. Linear stability analysis yields results similar to bulk spinodal decomposition, except that long, and possibly all, wavelength are stabilized. Decomposition into two phases is investigated by solving the diffusion equation for lamellar and cylindrical symmetry. For the lamellar geometry, typically observed for near-equal volume fractions, the diffusion equation does not yield wavelength selection criteria. These can be obtained if free energy minimization is assumed. For the cylindrical geometry, solutions for small volume fractions yield domain dimensions proportional to the deposition-rate dependent surface diffusion length.
\end{abstract}

\section{INTRODUCTION}

Interest in multicomponent films has been growing rapidly in recent years. The growth of multicomponent films forms the basis of such diverse technologies as compound semiconductors, magnetic recording, and optical devices. In some cases, the desired properties/ microstructures are the result of phase separation (e.g., magnetic recording media), while in others, phase separation or decomposition is an unintentional and often undesirable feature (e.g., InGaAsP). In bulk materials, the process of phase separation is well known and largely understood. ${ }^{1}$ These processes include ${ }^{1}$ spinodal decomposition, lamellar growth, second phase coarsening (Ostwald ripening), etc.

In many cases, the same types of phase separation processes that occur in the bulk also occur in films. However, they are usually complicated by two features unique to the film growth process: (1) deposition is occurring at a fixed, externally imposed rate and (2) atomic transport occurs preferentially along the advancing surface. The latter feature is associated with the fact that activation energies for surface diffusion are typically smaller than those for bulk diffusion by at least a factor of two and deposition is most often performed at low temperatures (relative to the melting point). This leads to a situation in which the majority of the microstructural development occurs at the advancing surface ${ }^{2}$ resulting in the freezing-in of the film structure once the film surface has advanced. Similar situations in which transport is limited to an advancing solid-solid interface have also received considerable attention (see, e.g., Ref. 3). One consequence of this type of picture is that the changes in the microstructure from the substrate to the surface represent the historical evolution of the microstructure on the surface.

In the present paper, we model the film microstructure as the history of the surface. This effectively reduces the dimensionality of the problem by one space dimension. We begin by deriving the surface chemical diffusion equation

\footnotetext{
a) Department of Nuclear Engineering.

b) Department of Physics.

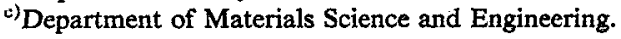

which is valid for finite film growth rates. This surface chemical diffusion equation is then employed in analyses of the two basic types of phase separation phenomena: spinodal decomposition and eutectic growth. In general, we find that these phenomena are very similar to the associated bulk problems, but at rates and size scales dictated by the deposition rate and surface diffusivity.

\section{SURFACE CHEMICAL DIFFUSION}

In order to describe the temporal evolution of the surface composition profile $c(x, y ; t)$, we' must account for Fickian diffusion along the surface and the fact that the surface is constantly being buried and incorporated into the bulk by the finite atomic deposition rate. We begin by considering the case of a nominally flat film, growing with velocity $v$ in the $+z$ direction as the result of a spatially and temporally uniform, normal flux of atoms. Assuming interdiffusivities $\widetilde{D}_{s}$ along the surface and $\widetilde{D}_{B}$ in the bulk, the equations describing the evolution of the compositional field $c(x, y, z ; t)$ in the frame of the advancing surface (in the $z$ direction) are

$$
\begin{aligned}
& \frac{\partial c}{\partial t}=\widetilde{D}_{s} \nabla_{s}^{2} c-\frac{\widetilde{D}_{B}}{\delta} \frac{\partial c}{\partial z}+v \frac{c_{0}}{\delta}-v \frac{c}{\delta} \quad(z=0: \text { surface }), \\
& \frac{\partial c}{\partial t}=\widetilde{D}_{B} \nabla^{2} c+v \frac{\partial c}{\partial z} \quad(z<0: \text { bulk }),
\end{aligned}
$$

where $\delta$ is the width of the surface diffusion layer (a few atomic spacings), $\nabla_{s}^{2}=\left.\left(\partial^{2} / \partial x^{2}+\partial^{2} / \partial y^{2}\right)\right|_{z=s}$, and where $z=s$ implies that the derivatives are to be evaluated on the surface. Throughout this paper we ignore effects associated with differences in atomic size between solute and solvent. Equation (1a) is the diffusion equation for the surface with advection into the bulk and with source and sink terms added to account for the fixed deposition rate at composition $c_{0}$ and incorporation into the bulk. The form of the advection term in Eq. (1a) makes use of the assumption that the rate at which atoms diffuse across the boundary separating the surface region and the bulk region is simply the bulk chemical interdiffusivity $\widetilde{D}_{B}$. In order to analytically solve Eq. (1) for the phase separation problems of 
interest, we will assume that the surface remains flat during the deposition, implying that $\left.\nabla_{s}^{2} \approx\left(\partial^{2} / \partial x^{2}+\partial^{2} / \partial y^{2}\right)\right|_{z=0}$. This assumption is not very severe as long as the amplitude of the surface height profile is small compared to the lateral extent of the domains of each phase.

For small $\widetilde{D}_{B}$, Eq. (1b) implies $c(x, y, z ; t) \approx c(x, y, 0 ; t$ $+z / v)$. In this limit, the bulk composition profile is simply a history of the surface composition profile. Assuming that the first term on the right-hand side of Eq. (1b) is small compared with the second, we obtain an expression for $\partial c / \partial z$ which may be inserted into Eq. (1a). This yields

$$
\begin{aligned}
\frac{\partial c}{\partial t} & \cong \frac{\left\{\widetilde{D}_{s} \nabla_{s}^{2} c+v\left[\left(c_{0}-c\right) / \delta\right]\right\}}{\left[1+\left(\widetilde{D}_{B} / v \delta\right)\right]}, \\
& \cong \widetilde{D}_{s} \nabla_{s}^{2} c+v \frac{\left(c_{0}-c\right)}{\delta} .
\end{aligned}
$$

The limiting form of the diffusion equation [Eq. 2(b)] was employed more than 30 years ago in a study of eutectoid decomposition. ${ }^{3}$ For common metal films (e.g., $\mathrm{Cu}$ ) grown under typical deposition conditions (e.g., room temperature, $v=1 \AA / \mathrm{s}), 1>\widetilde{D}_{B} / v \delta \sim 10^{-9}$. Therefore, $\widetilde{D}_{B}$ will be set identically to zero. This is known as the frozen bulk approximation. Given appropriate parameters and boundary conditions, the solution of this equation yields the film microstructure at all depths into the film.

\section{SPINODAL DECOMPOSITION}

For compositions corresponding to the solid solution region of the phase diagram perturbations to a uniform composition profile should decay away. For example, we can ask how the amplitude of an initial surface concentration perturbation of wavelength $\lambda$ decays with time. Inserting the initial sinusoidal surface concentration profile $c(x ; t$ $=0)=c_{0}+A \sin (2 \pi x / \lambda)$ into Eq. (2) and solving the resultant differential equation shows that the amplitude of the perturbation decays to the uniform composition $c_{0}$ as $c-c_{0}=\exp \left[-\left(4 \pi^{2} \widetilde{D}_{s} / \lambda^{2}+v / \delta\right) t\right]$. Therefore, surface concentration perturbations decay faster during deposition than in the static $(v=0)$ case. This is clearly attributable to the fact that the finite rate of deposition of the uniform composition $c_{0}$ buries the initially nonuniform composition profile.

In spinodal decomposition, the free energy versus composition curve exhibits two minima instead of just one. ${ }^{4}$ The presence of two minima indicate that for a range of compositions (between these two minima) two phases with the same structure but different compositions are in equilibrium. There exists a region between these two minima where the curvature of the free energy $\left(\partial^{2} f / \partial c^{2}\right)$ is less than zero (the locus of points at which $\partial^{2} f / \partial c^{2}=0$ at different temperatures defines the spinodal on the phase diagram). Since $\widetilde{D}=M \partial^{2} f / \partial c^{2}$ ( $M$ is a constant atomic mobility factor), the chemical interdiffusivity is negative within this region of the phase diagram and hence composition fluctuations grow (so called "up-hill diffusion"), resulting in a compositionally modulated material. If the composition fluctuations are sufficiently large that an atom

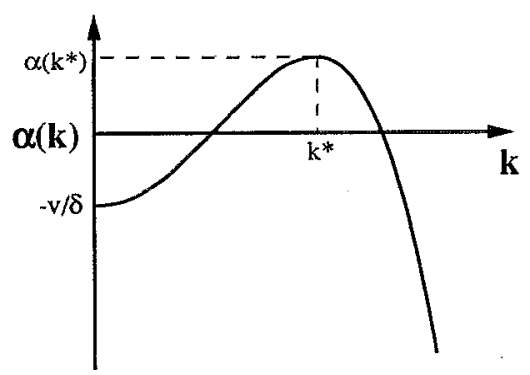

FIG. 1. The growth factor $\alpha$ for small-amplitude perturbations of wave number $k$ in spinodal decomposition. $k^{*}$ is the wave number of the fastest growing perturbation.

finds itself in a region where the composition is sufficiently different from the initial, uniform composition higher order gradient terms must be included in the diffusion equation. ${ }^{4}$ Including the next highest order gradient term, Eq. (2) is modified as

$$
\frac{\partial c}{\partial t}=\widetilde{D}_{s} \nabla_{s}^{2} c-2 M \beta \nabla_{s}^{4} c+v \frac{\left(c_{0}-c\right)}{\delta},
$$

where $\beta$ is a phenomenological parameter which may be determined experimentally.

For an initial sinusoidal surface composition perturbation, Eq. (3) is solved by a series of terms all of the form $c(k, t)-c_{0}=c(k, 0) e^{\alpha(k) t} \sin (k r)$, where $k$ is the wave number of the perturbation and $r$ is a position along the surface. The growth factor $\alpha(k)$ is given by: $\alpha(k)$ $=-M\left(\partial^{2} f / \partial c^{2}\right) k^{2}-2 M \beta k^{4}-v / \delta$ (see Fig. 1). $\alpha$ increases from its $k=0$ value $(-v / \delta)$ as $k^{2}$ and decays as $-k^{4}$ for large wave numbers (short wavelengths). Perturbations with wave numbers in the range - $\widetilde{D}_{s}-\left[\widetilde{D}^{2}\right.$ $-8 M \beta v / \delta]^{1 / 2}<4 M \beta k^{2}<-\vec{D}_{s}+\left[\vec{D}_{s}^{2}-8 M \beta v / \delta\right]^{1 / 2}$ grow while all other perturbations decay (recall $D_{s}<0$ within the spinodal). Perturbations of wave number $k^{*}=\left[\widetilde{D}_{s} / 4 M \beta\right]^{1 / 2}$ are maximally unstable and grow at a rate given by $\alpha\left(k^{*}\right)=\widetilde{D}_{s}^{2} / 8 M \beta-v / \delta$. When $v / \delta>\widetilde{D}_{s}^{2} /$ $8 M \beta$, all perturbations decay. These results for spinodal decomposition during film growth are essentially the same as in bulk materials, ${ }^{4}$ except for: (a) the stabilization of long wavelength, and possibly all, perturbations and (b) a slower overall rate at which the unstable modes grow. There is no difference, however, in the wavelength which will grow the fastest. However, the differences in the elastic boundary conditions appropriate for films and bulk materials may lead to some changes in this wavelength.

\section{EUTECTIC GROWTH}

\section{A. Lamellar geometry}

In many cases where the phase diagram is of the eutectic or eutectoid type, cooling through the eutectic (eutectoid) temperature into the two-phase region results in the formation of a microstructure consisting of a periodic arrangement of lamellae or rods. This type of phase separated microstructure can form in both purely solid state reactions such as in the growth of pearlite ${ }^{5}$ in steel ( $\mathrm{Fe}$ and 


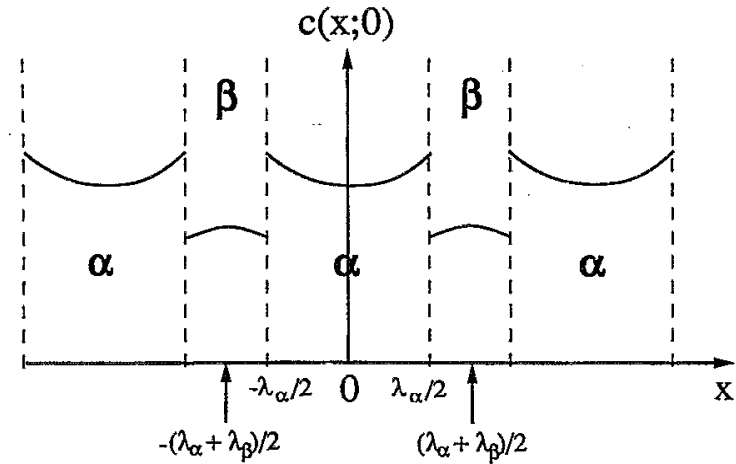

FIG. 2. The steady state surface composition profile from Eqs. (4) and (6). $\lambda_{\alpha}$ and $\lambda_{\beta}$ are the widths of the $\alpha$ and $\beta$ sizes of the eutectic lamellae, respectively.

$\mathrm{Fe}_{3} \mathrm{C}$ lamellae) and during solidification (e.g., $\mathrm{Al}-\mathrm{CuAl} 2$ lamellae ${ }^{6}$ ). Similarly, we expect that two-phase eutecticlike microstructures can also be formed during film growth. Our analysis of this problem is essentially identical to one performed by $\mathrm{Cahn}^{3}$ nearly 30 years ago for the solid state eutectoid transformation and is presented to set the stage for the less intuitive results, for the more important cylindrical case, presented below.

For a film grown with composition $c_{0}$ in the two-phase $(\alpha, \beta)$ region of a binary phase diagram, we denote the composition at the interface between the $\alpha$ and $\beta$ phases as $c_{\alpha}^{0}$ and $c_{\beta}^{0}$, respectively, where $c$ refers to the concentration of the majority element in the $\alpha$-phase, $A$. In the present analysis, we estimate the steady state composition profiles and lamellar spacings. The steady state composition profiles in the $\alpha$ and $\beta$ regions are obtained by setting $\partial c / \partial t=0$ in Eq. (2), yielding: $c_{\alpha}=A_{1} e^{x / \rho}+A_{2} e^{-x / \rho}$ and $c_{\beta}=B_{1} e^{x / \rho}$ $+B_{2} e^{-x / \rho}$, respectively, where $\rho=\left(\delta \widetilde{D}_{s} / v\right)^{1 / 2}$. The following boundary conditions are employed to determine the unknowns $A_{1}, A_{2}, B_{1}$, and $B_{2}$ and the sizes of the lamellae, $\lambda_{\alpha}$ and $\lambda_{\beta}$ : (1) $\partial c_{\alpha} / \partial x=0$ at $x=0$, (2) $\partial c_{\beta} / \partial x=0$ at $x$ $=\left(\lambda_{\alpha}+\lambda_{\beta}\right) / 2$, (3) $c_{\alpha}=c_{\alpha}^{0}$ at $x=\lambda_{\alpha} / 2$, (4) $c_{\beta}=c_{\beta}^{0}$ at $x$ $=\lambda_{\alpha} / 2$, and (5) $\partial c_{\alpha} / \partial x=\partial c_{\beta} / \partial x$ at $x=\lambda_{\alpha} / 2$.

The resultant compositions of the $\alpha$ and $\beta$ phases are given by

$$
\begin{aligned}
& c_{\alpha}(x)-c_{0}=\left(c_{\alpha}^{0}-c_{0}\right) \frac{\cosh (x / \rho)}{\cosh \left(\lambda_{\alpha} / 2 \rho\right)}, \\
& c_{\beta}(x)-c_{0}=\left(c_{\beta}^{0}-c_{0}\right) \frac{\cosh \left[\left\{2 x-\left(\lambda_{\alpha}+\lambda_{\beta}\right)\right\} / 2 \rho\right]}{\cosh \left(\lambda_{\beta} / 2 \rho\right)},
\end{aligned}
$$

where

$$
\frac{\lambda_{\alpha}}{2 \rho}=\tanh ^{-1}\left[-\left(\frac{c_{\beta}^{0}-c_{0}}{c_{\alpha}^{0}-c_{0}}\right) \tanh \left(\frac{\lambda_{\beta}}{2 p}\right)\right] .
$$

The spatial variations of the composition profile are shown in Fig. 2. For $\lambda_{\beta} / 2 \rho<1$ (i.e., large $\widetilde{D}_{s}$ or small $v$ limits) and for $\left(c_{\beta}^{0}-c_{0}\right) \lambda_{\beta} /\left[2 \rho\left(c_{\alpha}^{0}-c_{0}\right)\right]<1$, Eq. (5) reduces to the classical lever-rule result: $\lambda_{\alpha} / \lambda_{\beta}=-\left(c_{\beta}^{0}-c_{0}\right) /\left(c_{\alpha}^{0}-c_{0}\right)$. Since there were two unknown coefficients in the general solution to the differential equation for each phase and two unknown lamellar spacings and only five boundary conditions, the problem is underspecified. As a consequence there is no way to specify both $\lambda_{\alpha}$ and $\lambda_{\beta}$ (although their ratio is determined). This lack of wavelength selection is also characteristic of theoretical analyses describing eutectic solidification from the melt. ${ }^{7,8}$ Nevertheless, it is not unreasonable to expect that $\lambda$ is proportional to $\rho$. The mean composition within the individual $\alpha$ lamellae is

$$
\left\langle c_{\alpha}-c_{0}\right\rangle=\frac{2 \rho}{\lambda_{\alpha}}\left(c_{\alpha}^{0}-c_{0}\right) \tanh \left(\frac{\lambda_{\alpha}}{2 \rho}\right) .
$$

In the large surface diffusivity or small deposition rate limits the ratio of the concentration in the $\alpha$ lamellae $\left\langle c_{\alpha}-c_{0}\right\rangle$ to their nominal value $\left(c_{\alpha}^{0}-c_{0}\right)$ is $1-\left(\lambda_{\alpha}^{2} v\right) /$ $\left(12 \delta \widetilde{D}_{s}\right)$. On the other hand, in the large deposition rate or low diffusivity limit, this ratio is approximately $\left(4 \delta \widetilde{D}_{s} /\right.$ $\left.\lambda_{\alpha}^{2} v\right)^{1 / 2}$, which can be much different from unity (i.e., the phases become supersaturated). The mean composition within the $\beta$ lamellae is given by the same expression with $\beta$ substituted for $\alpha$. Using Eq. (2a) instead of (2b) would not affect these steady state results, but only the rate at which steady state is approached.

As indicated above, the solution of the diffusion equation does not provide a criterion for wavelength selection. Considering the thermodynamics of the system, it is expected that the excess free energy per atom due to supersaturation will increase and that the interfacial free energy decrease with the lamellar thickness. One possible approach is to postulate, as did Cahn, ${ }^{4}$ that the free energy of the system be minimized subject to the kinetic constraints. However; unlike in a eutectoid reaction ${ }^{4-8}$ where the velocity of the reaction front is a variable, in the present problem $v$ is determined by the deposition conditions. Following $\mathrm{Cahn},{ }^{4}$ it is assumed that the excess free energy of a supersàturated solution relative to $\alpha$ and $\beta$ in equilibrium is $K\left(c-c_{\alpha}^{0}\right)^{2} /\left(0.5-c_{\alpha}^{0}\right)^{2}$ for $\alpha$, and a similar expression for $\beta$, where $c$ is the composition and $K$ is an approximately temperature-independent constant. It is also assumed that $K$ is significantly greater than the free energy change upon formation of the terminal solutions from a uniform, amorphous phase. The lamellar thickness which minimizes the free energy, $\lambda_{0}$, satisfies:

$$
\lambda_{0}^{5}=960 \frac{\gamma \Omega}{K} \rho^{4}=960 \frac{\gamma \Omega}{K}\left(\frac{\widetilde{D}_{s} \delta}{v}\right)^{2},
$$

where $\gamma$ is the $\alpha-\beta$ interfacial free energy and $\Omega$ is an atomic volume. $\lambda_{0}$ is therefore proportional to $\left(\widetilde{D}_{s}\right)^{2 / 5}$.

The free-energy minimization determination of the wavelength $\lambda$ is consistent with experimental results in systems which are free to select the velocity of the reaction front. ${ }^{4}$ For film deposition, where the deposition velocity is imposed externally, the validity of such an approach has been questioned. In addition, Eq. (7) is based upon a particular functional form of the free energy of a supersaturated solution, which is difficult to verify experimentally. A different approach would be to set a limit on the degree of supersaturation of one of the phases: 


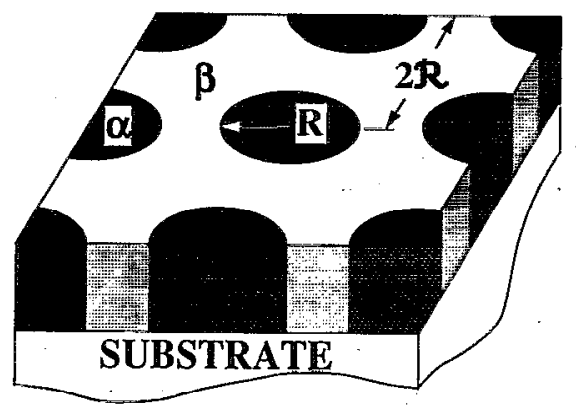

FIG. 3. Idealized cylindrical geometry assumed in the cylindrical phase separation analysis.

$$
c_{\alpha}^{0}-\epsilon_{\alpha}<c(x)<c_{\alpha}^{0}
$$

This is equivalent to assuming that the lamella of the opposite phase nucleates within a lamella whenever it exceeds a given supersaturation level, a process which sets an upper limit on the lamellar thickness. An implicit assumption in this simplified treatment of nucleation is that the size of a critical nucleus is much smaller than the lamellar thickness. Equation ( 8 ) yields the following condition:

$$
1-\frac{1}{\cosh \left(\lambda_{\alpha} / \rho\right)}<\frac{\epsilon_{\alpha}}{c_{\alpha}^{0}-c_{0}}
$$

As an example, if only a $5 \%$ supersaturation can be tolerated in the $\alpha$ phase which, at equilibrium, contains only $A$ atoms, an upper limit on $\lambda_{\alpha}$ is obtained: $\lambda_{\alpha} / \rho<0.47$.

\section{B. Cyllndrical geometry}

In many experimental studies of phase-separating films, the microstructure consists of columnar domains of different phases. We therefore solve Eq. (2) for cylindrical symmetry. The composition profile will be determined as a function of the domain radius, $R$, and the external parameters. In this geometry, we picture a regular array of cylindrical $\alpha$-domains of radius $R$ a distance $2 \Re$ apart, completely surrounded by the $\beta$ matrix (see Fig. 3 ). In order to simplify the problem, we focus on one $\alpha$ domain with the origin at its center, and apply the zero flux $(\partial c / \partial r=0)$ boundary condition at $r=\Re$. This assumption of cylindrical symmetry is valid provided that $\Re>R$, and therefore also $\left(c_{\alpha}^{0}-c_{\beta}^{0}\right)>\left(c_{0}-c_{\beta}^{0}\right)$, i.e., the average sample composition is close to that of the $\beta$ phase. The composition profile for a given domain radius, $R$, is derived, assuming stcady state, $\partial c / \partial t=0$. This yields

$$
c^{I}(r)=\left[c_{\alpha}^{0}(R)-c_{0}\right] \frac{I_{0}(r / \rho)}{I_{0}(R / \rho)}+c_{0}
$$

inside the cylindrical $\alpha$ domain, and

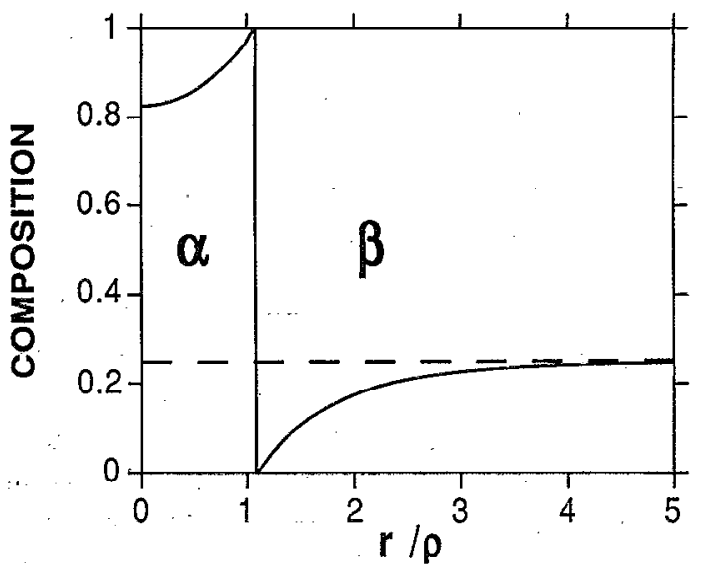

FIG. 4. Calculated composition profile for cylindrical $\alpha$ domain of radius $R / \rho=1.06$ embedded in a $\beta$ domain with $\Re=5$. The broken line denotes the average composition, $c_{0}=0.25$.

$$
\begin{aligned}
c^{0}(r)= & {\left[c_{\beta}^{0}(R)-c_{0}\right] } \\
& \times \frac{K_{0}(r / \rho) I_{1}(\Re / \rho)+I_{0}(r / \rho) K_{1}(\Re / \rho)}{K_{0}(R / \rho) I_{1}(\Re / \rho)+I_{0}(R / \rho) K_{1}(\Re / \rho)}+c_{0}
\end{aligned}
$$

outside the precipitate. $I_{n}$ and $K_{n}$ are modified Bessel functions of order $n$ and of the first and second kind, respectively. In the limit of large $\Re / \rho$, Eq. (11) reduces to

$$
c^{0}(r)=\left[c_{\beta}^{0}(R)-c_{0}\right] \frac{K_{0}(r / \rho)}{K_{0}(R / \rho)}+c_{0}
$$

Due to the Gibbs-Thompson effect, the equilibrium concentrations $c_{\alpha}^{0}$ and $c_{\beta}^{0}$ depend on the curvature of the interface and hence are functions of $R$ :

$$
\begin{aligned}
& {\left[1-c_{\alpha}^{0}(R)\right]=\left[1-c_{\alpha}^{0}\right] \exp \left(\frac{-\gamma \Omega}{R k_{B} T}\right),} \\
& c_{\beta}^{0}(R)=c_{\beta}^{0} \exp \left(\frac{\gamma \Omega}{R k_{B} T}\right) .
\end{aligned}
$$

For typical values of the interfacial energy $\left(2 \mathrm{~J} / \mathrm{m}^{2}\right)$, we find that the effect of the domain-boundary curvature is significantly only below $R<100 \AA$, so that, for larger $R$, the interfacial compositions can be assumed to be equal to the equilibrium values, $c_{\alpha}^{0}$ and $c_{\beta}^{0}$.

An additional constraint on $R$ and $\Re$ is obtained from mass balance

$$
\begin{aligned}
& \frac{c_{\alpha}^{0}(R)-c_{0}}{c_{0}-c_{\beta}^{0}(R)} \\
& \quad=\frac{\left[I_{1}(\Re / \rho) K_{1}(R / \rho)-K_{1}(\Re / \rho) I_{1}(R / \rho)\right] I_{0}(R / \rho)}{\left[K_{0}(R / \rho) I_{1}(\Re / \rho)+I_{0}(R / \rho) K_{1}(\Re / \rho)\right] I_{1}(R / \rho)}
\end{aligned}
$$

which for $\Re / \rho>1$ becomes

$$
\frac{c_{\alpha}^{0}(R)-c_{0}}{c_{0}-c_{\beta}^{0}(R)}=\frac{K_{1}(R / \rho) I_{0}(R / \rho)}{I_{1}(R / \rho) K_{0}(R / \rho)}
$$


For $c_{\alpha}^{0} \approx 1, c_{\beta}^{0} \approx 0$, and $\Re / \rho \geqslant 4$, Eq. (14) yields the following approximate values for $R / \rho: 0.83$ for $c_{0}=0.2$, and 0.43 for $c_{0}=0.1$. A composition profile calculated for $c_{0}=0.25$ and $\Re / \rho=5$ is shown in Fig. 4.

We thus obtain that, under the assumptions $R<\Re$ and $\Re>\rho$, the two-dimensional analysis yields a steady state domain radius, proportional to $\rho=\left(\widetilde{D}_{s} \delta / v\right)^{1 / 2}$, which is determined by the diffusion equation alone. As discussed before, the assumed geometry, and therefore this solution, is applicable when the left-hand side of Eq. (14) is sufficiently greater than 1 . Similar algebra could be used in the lamellar case, however, lamellae are observed experimentally only for similar $\alpha$ and $\beta$ volume fractions. Figure 4 shows significant supersaturation of both phases, which could lead to the precipitation of new domains, leading to a reduction in $\Re$, and possibly invalidating the analysis. In this case, the limit on supersaturation could determine the scale of phase separation, similar to our analysis of the lamellar geometry.

Thompson ${ }^{9}$ has analyzed the process of island coarsening on a substrate by adatom diffusion, and Atwater and Yang $^{10}$ have extended the theory to include addition or removal of atoms due to deposition or sputtering, respectively. The present treatment differs from Refs. 9 and 10 as follows: (1) it accounts for supersaturation within the domains, not only in the area surrounding them; (2) when capillary effects are negligible due to large domain radii, the steady state domain size is determined by the diffusion process alone; (3) the diffusion time is finite and determined by the deposition rate and diffusivities. As a result of (2) and (3), when capillary effects are negligible, a steady state is obtained which is independent of the initial conditions.

The present results suggest that the steady state $\alpha$ domain diameter is proportional to $\rho=\left(\widetilde{D}_{s} \delta / v\right)^{1 / 2}$. Since the only parameter which varies with temperature is $\widetilde{D}_{s}$ the domain diameter should have the same temperature dependence as $\left(\widetilde{D}_{s}\right)^{1 / 2}$. This result was successfully used to relate the surface diffusivity to domain size in Ref. 11.

\section{CONCLUSION}

Spinodal decomposition and two-phase coarsening during film deposition have been analyzed in the limit of negligible bulk diffusion. Linear stability analysis yield results similar to bulk spinodal decomposition, except that long, and possibly all, wavelengths are stabilized. In the growth of a film within a phase separating region of a phase diagram eutectic/eutectoid-like lamellar geometries can occur. For the lamellar geometry and near-equal volume fractions of the two phases, the diffusion equation alone does not lead to wavelength selection. When the volume fraction of one of the phases is low, cylindrical or rod-like eutectoid phase separation may occur. In this small volume fraction regime, the diameter of cylindrical domains may be determined by the diffusion equation alone, and is proportional to $\rho=\left(\widetilde{D}_{s} \delta / v\right)^{1 / 2}$.

\section{ACKNOWLEDGMENTS}

This research was supported by the National Science Foundation under Grant No. DMR-8820285 (M.A.), and by the U.S. Department of Energy under Grant No. DOE DE-FG-02-85ER54189 (D.A.K.) and by the U.S. Air Force Office of Scientific Research under Grant No. AFOSR-90-0112 (D.J.S.). The authors wish to thank Professor A. Patera and Professor F. Spaepen for stimulating discussions and helpful comments.

${ }^{1}$ R. W. Cahn and P. Haasen, Physical Metallurgy (North-Holland, Amsterdam, 1983).

${ }^{2}$ D. J. Srolovitz, A. Mazor, and B. G. Bukiet, J. Vac. Sci. Technol. A 6, 2371 (1988)

${ }^{3}$ J. W. Cahn, Acta Metall. 7, 18 (1959).

${ }^{4} J$ J. Cahn, Trans. AIME 242, 166 (1968); Acta Metall. 9, 795 (1961).

${ }^{5}$ V. Zackay and H. Aaronson, Decomposition of Austenite by Diffusional Processes (Interscience, New York, 1962).

${ }^{6}$ L. D. Graham and R. W. Kraft, Trans. AIME 236, 94 (1966).

${ }^{7}$ K. A. Jackson and J. D. Hunt, Trans. AIME 236, 1139 (1966).

${ }^{8}$ D. A. Kessler and H. Levine, J. Cryst. Growth 94, 871 (1989).

${ }^{9}$ C. V. Thompson, Acta Metall. 36, 2929 (1988).

${ }^{10}$ H. A. Atwater and C. M. Yang, J. Appl. Phys. 67, 6202 (1990).

${ }^{11}$ (a) M. Atzmon, C. D. Adams, Y.-T. Cheng, and D. J. Srolovitz, Mater. Res. Soc. Symp. Proc. 202, 143 (1991). (b) C. D. Adams, M. Atzmon, Y.-T. Cheng, and D. J. Srolovitz, J. Mater. Res. 7, 653 (1992). 